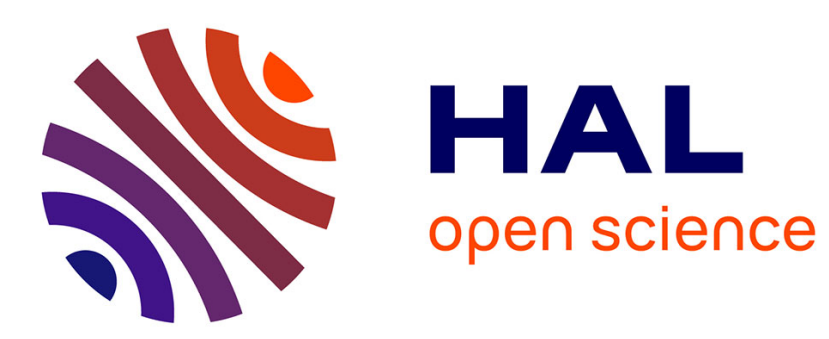

\title{
Stress-velocity boundary condition and the localization of deformation
}

\author{
Y. Leroy, B. Bonvalot, A. Molinari
}

\section{To cite this version:}

Y. Leroy, B. Bonvalot, A. Molinari. Stress-velocity boundary condition and the localization of deformation. Journal de Physique IV Proceedings, 1994, 04 (C8), pp.C8-429-C8-434. 10.1051/jp4:1994866 . jpa-00253427

\section{HAL Id: jpa-00253427 https://hal.science/jpa-00253427}

Submitted on 1 Jan 1994

HAL is a multi-disciplinary open access archive for the deposit and dissemination of scientific research documents, whether they are published or not. The documents may come from teaching and research institutions in France or abroad, or from public or private research centers.
L'archive ouverte pluridisciplinaire HAL, est destinée au dépôt et à la diffusion de documents scientifiques de niveau recherche, publiés ou non, émanant des établissements d'enseignement et de recherche français ou étrangers, des laboratoires publics ou privés. 


\title{
Stress-velocity boundary condition and the localization of deformation
}

\author{
Y.M. Leroy(1), B. Bonvalot and A. Molinari
}

Laboratoire de Physique et Mécanique des Matériaux, URA 1215 du CNRS, Université de Metz, Ile du Saulcy, 57045 Metz cedex 1, France

\begin{abstract}
résumé: On étudie, au moyen de la méthode des éléments finis, l'influence des conditions aux limites mixtes ( relation linéaire entre vitesse et contrainte ) sur la stabilité des états stationnaires et sur la localisation de la déformation. Cette condition mixte, qui prévaut dans la barre de Kolsky en torsion, est notamment dépendante de la géométrie des barres et de l'échantillon. On montre que le coefficient qui apparait dans la condition mixte contrôle l'évolution d'un état stationnaire instable vers un état stationnaire stable jouant le rôle d'attracteur. Ce même coefficient joue un rôle mineur sur la déformation à la localisation, cette dernière étant contrôlée par la quantité d'énergie dissipée au cours de l'essai. Toutefois, la vitesse à laquelle la contrainte chute durant la phase de localisation rapide, est influencée par la condition mixte.
\end{abstract}

abstract: The influence of the mixed mechanical boundary condition, defined by a linear relation between velocity at the boundary and stress, on the non-linear stability of steady states and on the localization of the deformation is investigated by numerical means. This mixed boundary condition is known to prevail during a torsional Kolsky bar test and depends on the geometry of the incident bar and of the specimen. It is shown that the coefficient which enters the mixed condition governs the evolution of the flow from a perturbed, unstable steady state towards a stable attractor. The same coefficient is found to have little influence on the nominal strain at initiation of the localization which appears to be governed by the total energy dissipated. However, the rate at which the stress drops during the development of the localization is affected by the mixed boundary condition.

\section{INTRODUCTION}

The torsional Kolsky bar test is widely used to study materials submitted to high rates of strain and to assess the material tendency to fail by localization of the deformation [1]. The design of the testing device [2] is such that a series of reflexions of the incident wave occurs within the confine of the specimen, 
a thin-walled cylinder, and renders the state of stress approximately uniform during the main part of the test. Consequently, the material properties at high rate of strain can be measured with little inference of inertia effects. It has not yet been fully appreciated that, as a consequence of the test design, the mechanical boundary condition which prevails during the measurements is mixed between the velocity and the stress. The velocity and the stress are linearly related with a slope, referred to in what follows as the boundary coefficient, determined by the geometry of the incident bar and of the specimen [3]. The influence of this mixed boundary condition on the linear stability of steady states has been studied by Leroy and Molinari [3]. This contribution is an extension of that work and is concerned with the role of the mixed boundary condition on the non-linear stability of steady states and on the localization of the deformation during a dynamic test.

The contents of this paper are as follows. The governing equations for the 1-D shear flow of a thermo-viscoplastic material with mixed boundary condition between the velocity and the stress are presented in Section 2. This plane-Couette flow is the model adopted to study the temperature and the deformation distributions on a specimen tested with a torsional Kolsky bar. The main results of Leroy and Molinari's work [3], concerning the linear stability of plane-Couette flow steady states, are commented in Section 3. Section 4 extends the linear stability analysis: finite-element simulations of the evolution with time of the temperature and of the deformation are reported to assess the non-linear stability of the steady states. The mixed boundary condition is found to be responsible for the selection of the stable attractor which determines the evolution of the flow starting from a perturbed, unstable steady state. These results on the linear and non-linear stability of steady states are compared with those on the localization of the deformation presented in Section 5. There, the initial conditions of the numerical simulations are those at the beginning of the dynamic test. The work-hardening properties of the material and typical defects in specimen geometry are accounted for. It is found that the boundary coefficient has little influence on the nominal deformation at localization, if the same values of the shear stress and of the strain rate are obtained prior to localization in all simulations. However, we observe a strong influence of the boundary coefficient on the rate at which the specimen bearing capacity drops during the development of the localized deformation.

\section{GOVERNING EQUATIONS}

The problem chosen to model the cylindrical specimen tested with a torsional Kolsky bar is one of a layer of finite thickness and infinite extent sustaining an overall simple shear mode of deformation. This layer, shown in Fig. 1, is composed of a thermo-viscoplastic material. The elastic properties of the material as well as the inertia effects, the latter having a role to play during the early part of the dynamic test as well as during the localization of the deformation, are disregarded.

The problem is formulated in one dimension, disregarding possible variations in shear band thickness that were revealed by a 2-D linear stability analysis [4]. The equilibrium condition dictates that the shear stress $\tau$ is uniform over the specimen and is thus a function of time only. The evolution of the temperature $\theta$ in time and space is governed by the heat equation written as:

$$
\dot{\theta}-\theta_{, y y}=k \tau v_{, y},
$$

in which the velocity in the shear flow is denoted by $v$. The dot and the comma stands for partial differentiation with respect to time and to the variable in subscript, respectively. The $y$-axis originates at the centre of and is perpendicular to the shear zone. All lengths are scaled by the half-width of the layer $d$. The time variable has been non-dimensionalized with the characteristic time for heat conduction $t_{C}$ defined by $\rho C d^{2} / \lambda$ in which $\rho, C$ and $\lambda$ are the material density, the specific heat and the thermal conductivity, respectively. The dimensionless number $k$, which typically has a value of 0.4 , is not the 
Taylor-Quinney coefficient but the ratio of a second characteristic time $t_{Q}$ over $t_{C}$. This second time scale $t_{Q}$ is obtained from the heat equation under stationary conditions; its precise definition is found in the dimensional analysis of Leroy and Molinari [3] which we have adopted here.

We now turn to the thermo-viscoplastic constitutive model. In the absence of elastic deformation, the velocity gradient is related to the shear stress via the following non-Newtonian law:

$$
\tau=\exp (-\beta \theta) f(\gamma)(v, y)^{m}
$$

in which $m$ is the strain rate exponent and $f$ a function of the accumulated deformation $\gamma$. This function $f$ has the constant value of 1 during the analysis of the steady states and corresponds to a power law in the analysis of the localization. In eq. (2), the decrease in stress with increasing temperature has been modeled by an exponential function which is a first-order approximation of the Arrhenius law. The coefficient $\beta$ is the thermal softening coefficient and the temperature is normalized by the external, reference temperature.

Concerning the boundary conditions, first note that the solutions of the transient problem as well as the steady state solutions are symmetric with respect to the centre of the shear zone allowing us to restrict the analysis to the upper part of the layer. Consequently, the velocity and the heat flux at the origin $y=0$ are set to 0 . We impose an isothermal condition at the boundary $y=1$ by setting the temperature there to a reference value of unity: $\left.\theta\right|_{y=1}=1$.

The boundary condition central to this paper is the mechanical boundary condition at $y=1$. The wave analysis of the torsional Kolsky bar test [3] indicates that this condition is mixed between the velocity and stress and reads:

$$
v-\phi \tau=-\phi \tilde{\tau} \quad \text { at } \quad y=1 .
$$

The stress $\tilde{\tau}$ is the amplitude of the incident shear pulse and the boundary coefficient $\phi$ is given by the expression:

$$
\phi=-\frac{t_{C} \tau_{R}}{d} \frac{8 r^{2} h}{R^{4} \sqrt{\mu \rho_{B}}}
$$

in which the first term on the right-hand side results from dimensional analysis ( $\tau_{R}$ is a reference stress). The second term on the right-hand side of eq. (4) shows the dependence of $\phi$ on the geometry and material properties of the incident bar and on the geometry of the specimen. The parameters $\rho_{B}, \mu$ and $R$ are the bar material density, its modulus of elasticity in shear and its radius, respectively. The characteristics of the specimen which enters the expression for $\phi$ are the radius $r$ of the cylinder and the thickness $h$ of the thin wall. By changing the design of the incident bar or of the specimen, the value of $\phi$ could be modified. Note also that the values for $\phi$ of 0 and $-\infty$ correspond to constant velocity and constant stress at the boundary, respectively.

\section{LINEAR STABILITY OF STEADY STATES}

Analytical expressions for the steady state solutions have been presented by various authors and a review of the early work on this subject is found in [3]. The structure of these solutions is depicted in Fig. 2 in a space spanned by the shear stress and the velocity at the boundary. The curve presented has a maximum in shear stress which is attained for a velocity $v\left(\theta^{*}\right)$ function of the critical temperature $\theta^{*}$ at the centre of the specimen, the latter being approximated by

$$
\theta^{*} \simeq 1+\frac{4}{3} \frac{m}{\beta}
$$


The ratio $m / \beta$ of the strain rate exponent over the temperature thermal coefficient has a value of approximately $1 / 30$ for structural steels. Therefore, the temperature $\theta^{*}$ is close to unity and the steady states relevant to a dynamic test are to the right of the maximum in view of the high temperature reached during localization. Steady states to the left of the maximum on the curve of Fig. 2 are said to be on the subcritical branch and the ones to the right are referred to as the supercritical states. This denomination originates from the linear stability of the steady states with a prescribed stress at the boundary: the subcritical and supercritical states are stable and unstable, respectively ([5], [3]). However, all steady states are shown to be stable, if the velocity is prescribed at the boundary. The stability conditions for the mixed boundary condition of interest to this paper are more complex. It is found that the conditions of neutral stability are met on points of the $(\tau, v)$ curve where the slope equals the inverse of the boundary coefficient $\phi$. Depending on the value of the parameter $\phi$ and the slope of this curve, a "window" of instability could be present on the supercritical branch. This case is illustrated in Fig. 2: the steady states between points 1 and 2 are unstable for the value of $\phi$ adopted. For example, the steady state at point 3 is unstable and a perturbation results in a flow which departs from steady conditions. The evolution away from the unstable steady state will be along the path defined by the straight line intersecting the steady state curve at point 3 , since the mixed boundary condition (3) is always satisfied. The velocity at the boundary could either increase or decrease until one of the two steady states indicated by points $3 \mathrm{a}$ and $3 \mathrm{~b}$ are encountered. These two steady states are subcritical and supercritical, respectively; both are linearly stable and thus could act as attractor.

This interpretation of the flow evolution of an unstable steady state towards an attractor does not provide any idea on the time necessary for the change in flow to occur. It is the objective of the next section to answer that question by presenting results concerning the non-linear stability of steady states.

\section{NON-LINEAR STABILITY OF STEADY STATES}

We wish to explore the evolution in time of the unstable steady states. No analytical solution is available and we resort to a finite-element scheme. The unknowns of the numerical analysis are the temperature nodal values and the shear stress which is constant over the whole specimen. Typical results are presented in Fig.3.

The initial conditions of the simulations are those of a steady state such as point 3 on the steady state curve of Fig. 2. The steady states are perturbed by increasing or decreasing the temperature in the shear zone by a relative amount of $1 \%$. It is found that the shear flow is attracted by the subcritical or the supercritical steady state ( such as point $3 a$ or $3 b$ ) depending on whether the initial perturbation leads to a decrease or an increase of the temperature, respectively. Note from Fig. 3 that the time required for the flow to change from an unstable steady state to a stable steady state decreases for larger absolute values of the boundary coefficient $\phi$. This coefficient determines also the position of the steady state to be reached on the $(\tau, v)$ curve in Fig. 2. This influence is clearly seen in Fig. 3 for the steady states reached on the supercritical branch. For example, for $\phi=-25$, the supercritical attractor is reached after an increase in velocity at the boundary of $50 \%$ and a time lapse of more than $7 t_{C}$. This increase is of the order of 400 $\%$ and occurs over a time of approximately $3 t_{C}$ if $\phi$ is set to -40 .

The conclusion of this non-linear stability analysis is that the larger the absolute value of $\phi$, the faster the instability develops and the larger the velocity of the stable attractor. It remains now to question whether the boundary coefficient will have a similar influence on the localization of the deformation and more precisely if a larger magnitude of $\phi$ is conducive to a faster localization.

\section{LOCALIZATION OF THE DEFORMATION IN SHEAR BANDS}

The initial conditions of the numerical simulations are now those of the dynamic test. A small 
perturbation in temperature is introduced to model the geometric defects that are typically found on the surface of the specimens [6]. This equivalence between temperature and geometric defects relies on the assumption of adiabaticity which is satisfied before the initation of the localization. Indeed, the time required for localization is small compared with the characteristic time $t_{C}$ and heat conduction plays only a role during localization on the length scale of the developing shear band. This is at a difference with the preceding analysis of the steady states. Note that the work-hardening properties of the material are also accounted for. The material parameters chosen are those of the HY-100 reported by Marchand and Duffy [2].

Before embarking on the analysis of the finite-element results, we use again Fig. 2 to illustrate the evolution of the flow. The initial conditions of the dynamic test correspond to a zero velocity at the boundary and the shear stress equal to a constant $\tilde{\tau}$, which is larger than the maximum of the steady state curve. Shortly after the beginning of the test, the mixed boundary condition dictates the relation between the velocity and the stress and a single steady state can act as an attractor, if it is found to be stable (point 4 in Fig. 2).

We now turn to the results of the finite-element simulations obtained for the three values of $\phi$ of $\phi_{K}, \phi_{K} / 30$ and $30 \phi_{K}$, Fig. 4. In this figure, the shear stress is presented as a function of the accumulated strain for various values of the boundary coefficient $\phi$ and stress $\tilde{\tau}$. For every value of $\phi$, the stress $\tilde{\tau}$ is chosen such that the nearly homogeneous strain rate prior to localization has a value of approximately $1500 \mathrm{~s}^{-1}$. We observe from Fig. 4, that the stress first increases due to the work-hardening properties of the material, then remains constant during the main part of the simulations. The sharp drop observed at the end of a simulation corresponds to the initiation of the localization of the deformation. Note that for a boundary coefficient of $\phi_{K}$, the nominal strain at localization $(0.60)$ and the time required for localization to occur $(370 \mu s)$ are comparable with the experimental values reported by Marchand and Duffy. These simulations reveal that the nominal strain at initiation of the localization is not sensitive to the boundary coefficient $\phi$. This observation indicates that the total energy dissipated is more relevant for the initation of the localization than the precise nature of the boundary condition. However, the slope of the $(\tau, v)$ curves beyond the initiation stage is determined by $\phi$, as it is clearly seen in Fig. 4 . Thus, the development of the localized deformation is strongly affected by the mixed boundary condition.

\section{REFERENCES}

[1] Marchand A. and Duffy J., J. Mech. Phys. Solids (1988) 36251.

[2] Kolsky H. Proc. phys. Soc. Lond. Ser B (1949) 62676.

[3] Leroy Y.M. and Molinari A., J. Mech. Phys. Solids (1992) 40 181-212.

[4] Molinari A. and Leroy Y.M., C. r. hebd. Séanc. Acad. Sci. Paris Sé. II (1991) 313 7-13.

[5] Chen H.T., Douglas A.S. and Malek-Madani R., Quarterly of Applied Mathematics (1989) 47 247-262.

[6] Molinari A. and Clifton R.J., J. Applied Mechanics (1987) 36251 


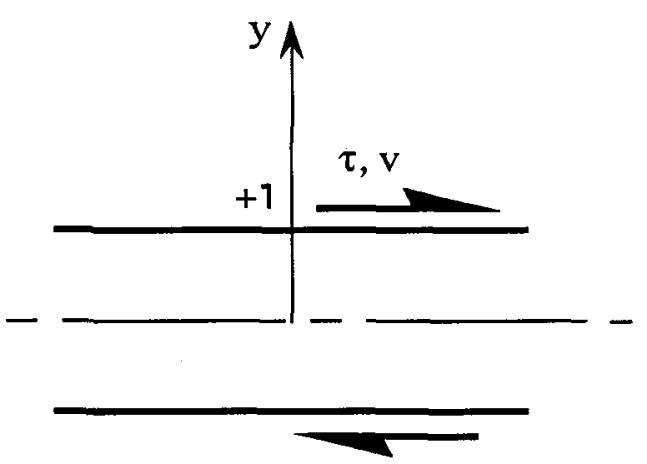

Fig. 1: Model problem : a layer of infinite extent sustains an overall simple shear with mixed boundary condition between the shear stress and the velocity.

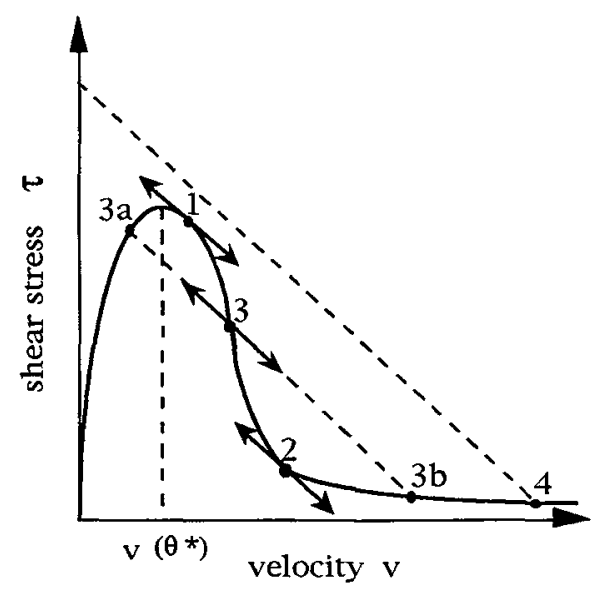

Fig. 2: Schematic representation of the steady state solutions.

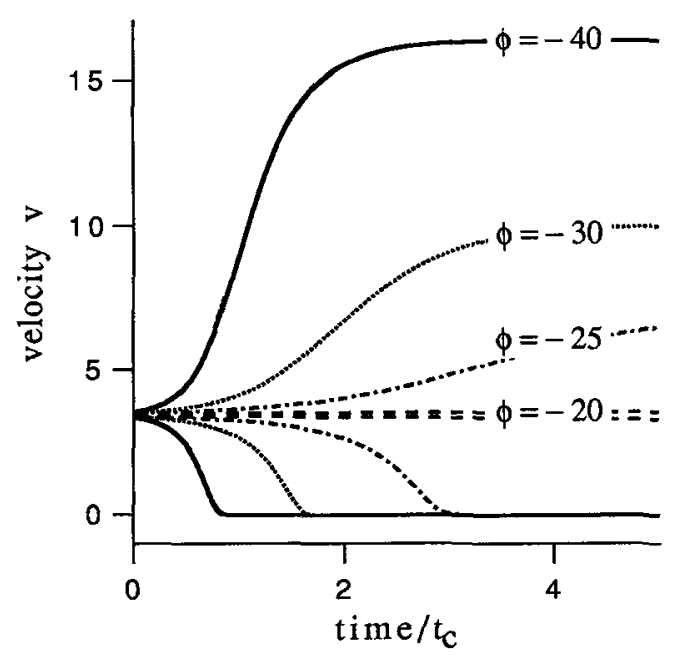

Fig. 3: Non-linear stability analysis of the steady states. Influence of the boundary coefficient $\phi$.

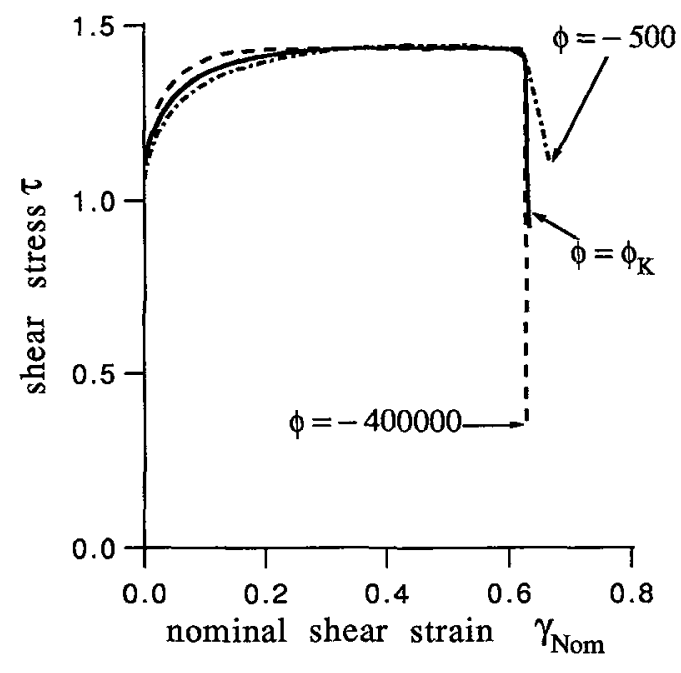

Fig. 4: Evolution of the stress with respect to the nominal shear strain for the initial temperature profile:

$$
\theta(y)=1.125-0.125|\mathrm{y}|
$$

